\title{
RANKING FOUR AND FIVE STAR HOTELS BASED ON CUSTOMER SATISFACTION WITH TEXT MINING ALGORITHMS: A SURVEY RESEARCH ON BANGKOK HOTELS
}

\author{
Farhad Safiri and Seyed Mohammad Jafar Jalali \\ Department of information Technology Management
}

\begin{abstract}
Nowadays, Tourism is one of economics' Foundation of each country and it is injected money to GNP of each country directly hence having a good environment is a mandatory plan for upgrading mentioned industry. One of aspect of tourism is related to hospitality and hotels which means how we can provide best services and hospitalities for visitor and tourist. In the latest decades there are many researches in this case that show which factor in hospitality is important and which one isn't important and how can we improve hospitality. The big concern of each tourist is choosing right hotel that it's started since they make first decision to have a trip to abroad so they face many hotels with many facilities also after reading related comment for each hotel they can't make a right decision in this regard. In this research our goal is to show which hotel is good and why and provide an efficient and trustful ranking for hotels at the specific district with text mining algorithm which is helped people to have best and right choice in their trip. Text mining algorithm can find hidden pattern in each text and help us to find best point for each hotel and make a suggestion system for each case especially in hospitality.
\end{abstract}

Keywords: Keywords: hospitality, hotel, text mining , algorithm

\section{INTRODUCTION}

Hotel sector is one of most important part of tourism industry and it's going to be top priority for each country which wants to have more profit in tourism industry and raising country GNP. Customer's satisfactions and comments are very important to each hotel to improve their services, one the other hand web2 is a tool for reviewing customer's comments and help customer to have the best choose among on many hotels. A popular form of Web 2.0 applications includes online review websites. These allow users to effectively disseminate their postpurchase experiences of products and services to the online communities at little cost.

These comment have some side effects for new customer, old customer and hotel owner which means some comments can decrease the level of a specific hotel or increase meanwhile help hotels to fix their weakness and improve their facilities and services to customer.
Social media and customer produce content on the internet rapid to grow and influence the hospitality

industry(Browninget al., 2013; Xiang and Gretzel, 2010). The scale growth of these data has inspired the new way to understanding the large amount of data(Wood et al., 2013; George et al., 2014).

Online booking system provide rating from travels in two parts(Buhalis \& Law, 2008) : first one is related to star rating from one to five for overall and for each specific textual description .Second one is related to sentiment comments for each hotel and it's very vary across many hotels(Kirk, 1995; Namasivayam, Enz, \& Siguaw, 2000).

Hotel guest experience and review have been an interesting topic for many years because it contains a widely range of comment that related to customer royalty, repeat purchase, word of mouth and 
ultimately higher profitability(Oh and Parks,1997) hence this paper is shown how to use online review for better choosing a good hotel and why we should choose a specific hotel rather than other hotel.

As Meyer and Schwager ( 2007 ) point out, customer experience is more related in the current society, it means consumer have more choices for selection a hotel based on previous experiences (e.g., Clemes et al., 2011;Cronin et al., 2000) which had written by other customers(e.g., Chen and Chen, 2010; Zins, 2002). Although most of studies have been focused on service quality, staff behavior, location, cleanness and top attributes which are used for each hotel that reflexed on customer comments and satisfactions but in previous research it hasn't been discovered and separate comments based on negative and positive comments that called sentiment analysis.

\section{LITERATURE REVIEW}

\section{Hotel guest experience and satisfaction :}

Hotel guest satisfactions and comments are a complex experience based on quality of hospitality services. Studies of hotel guest satisfactions have been started from early 1970s but new definition has been raise and discovered the replaced by old definition. Hunt (1975) point out, satisfactions is a value for evaluation service on each hotel and prove that it's same as thing that supposed to be while others (e.g.,Oliver,1981) define consumer satisfactions can be as an emotional response to use a service or product the estimate the quality and rate of service .

Oh and parks (1997) indicate that satisficcation understanding and efficient processes as well as other psychological influence also customer experience has two sides : satisfaction/dissatisfaction for a product that is purchased with evaluation (Engel et al., 1990).

Form managerial points of view, it's necessary to understand the view and main content of hotel guest satisfaction .For example, it has been imaged that hotel product consist of many levels such as core level ( hotel room ) that directly related to what customer purchase meanwhile hotel product also includes facilities, staffs, view and location .It means how to customer can get good from the purchase from all aspect such as front desk, staff behavior, breakfast, room service and etc. that belong to core product and various value-added products and services(Kotler et al., 2006).

\section{Online Booking website Comments :}

Travelers are classified into one and five group .It appears on all Online booking website .These profiles are couple, friend, family, friend and solo (Dolnicar, 2002;O'Connor, 2008) while these groups have different purpose and exception for traveling (Ariffin \& Maghzi, 2012) .

Travelers from different group have various perception and view from hotel product and services such as cleanliness ,safety, value for money and location (Atkinson, 1988; Lewis, 1985).

As we mentioned in the last paragraph, each group has a different view for selection a hotel, for example business travelers have many concern about location of hotel and availability of internet rather than room price (Bulchand-Gidumal, Meli an-Gonz alez, \& L opez-Valc arcel, 2011; Rivers, Toh, \& Alaoui, 1991). satisfaction of peoples who travel as couple is largely different with a business travel and affect by many factors that related to hotel, location and city (Lee,Huang, \& Chen, 2010) also family and friends travelers put their focus more on safety and security compare with solo travelers(Lai \& Graefe, 2000). All rates are provided by travelers in different profile with different view (Poston, 2008) also they commonly want to post their experience meanwhile a user who booked a hotel and had an experience of a specific hotel share his/her experience with other people regarding hotel that was booked (Gretzel, Yoo, \& Purifoy, 2007).

\section{Text mining:}

Text mining is a Technique for extraction data and hidden pattern for a text and it consists of some method for doing and find related pattern.

- Association rule

- Decision tree

- Classification 
- Clustering

\section{Bangkok City:}

Bangkok city is capital of Thailand and it has been in one of tourism distract in the world and it's getting to grow to be a top destination for tourist also city provides many top four and five star hotels for this purpose, on the other hand some attractive place encourage people to have a trip for one time to this attractive and beautiful city in the specific region.

\section{METHOD}

\section{Data Source:}

All of this paper data are gathered from booking.com website due to some reasons.booking.com is a website for booking hotel around the world also they provide exclusive system for putting customer review. Booking.com provides comment in tow aspects: negative and positive comments that help to customer to have better understanding of situation of hotel and make better decision for choosing good hotel. The last reason is that booking.com provides a rating from 0 to 10 for 6 specific attribute and product of hotel such as cleanliness, Location, staff, Free Wi-Fi , Comfort, Value for Money and also have overall rating for hotel .

\section{Data Collection and Analysis :}

For this section, Bangkok is selected as a tourism destination and all data are extracted form booking.com website by a python program that developed and written exclusively for this purpose. In first phase all reviews are extract for four and five star hotels and divided into two parts: negative and positive comments then all data are cleaned and preprocessed by $\mathrm{R}$ program for removing noise and none English comments that increase error. In third phase we provide word frequency for finding top attributes for customer that was important and based on result and clean our data from un-necessary attributes and data. In final phase based on latest data we provide a ranking and weight for all four and five start hotels in Bangkok city in two different sides : positive and negative .

Table 1 Ranking Four Star Hotels .

\begin{tabular}{|c|l|c|}
\hline Rank By Medium Weight & Hotel & Rank \\
\hline 0.642857143 & TheDavisBangkok & 1 \\
\hline 0.633333333 & FuramaSilomHotel & 2 \\
\hline 0.616666667 & ApartmentsSomersetL & 3 \\
\hline 0.607142857 & NouvoCityHotel & 4 \\
\hline 0.602941176 & MajesticGrandeHotel & 5 \\
\hline 0.575757576 & GalleriaSukhumvit10BangkokbyCompassHospitality & 7 \\
\hline 0.558139535 & CasaNithra & 8 \\
\hline 0.552631579 & EvergreenPlaceBangkok & 9 \\
\hline 0.548387097 & AloftBangkok-Sukhumvit11 & 10 \\
\hline 0.536585366 & GrandSwissSukhumvit & 11 \\
\hline 0.534482759 & ApartmentsSomersetS & 12 \\
\hline 0.527777778 & CentrePointSukhumvi & 13 \\
\hline 0.52 & ApartmentsSomersetP & 14 \\
\hline 0.514705882 & DynastyGrandeHotel & 15 \\
\hline 0.5 & CentrePointSilom & 16 \\
\hline 0.5 & ParkPlazaSukhumvit & 17 \\
\hline 0.4875 & CentrePointChidlom & 18 \\
\hline 0.483870968 & MercureBangkokSiam & 19 \\
\hline 0.47 & USukhumvitBangkok & 20 \\
\hline 0.465116279 & AdelphiSuitesBangkok & 21 \\
\hline 0.455882353 & BestWesternPremier & 22 \\
\hline 0.445945946 & ApartmentsLohasSUITESSUKHUMVITbySUPERHOTEL & 23 \\
\hline 0.439393939 & HolidayInnExpressBangkokSiam & 6 \\
\hline 0.435897436 & ChatriumResidenceSathonBangkok & 7 \\
\hline
\end{tabular}




\begin{tabular}{|c|l|l|}
\hline 0.42 & BaiyokeSkyHotel & 25 \\
\hline 0.402777778 & RembrandtHotelBangkok & 26 \\
\hline 0.393939394 & PresidentSolitaireHotel\&Spa & 27 \\
\hline 0.390625 & GMSuites & 28 \\
\hline 0.39 & RivaSuryaBangkok & 29 \\
\hline 0.384615385 & FourPointsbySheratonBangkok,Sukhumvit15 & 31 \\
\hline 0.381578947 & BelAireBangkok & 32 \\
\hline 0.368421053 & NovotelBangkokPloen & 33 \\
\hline 0.357142857 & CondoHotelTonsonResidence & 34 \\
\hline 0.352941176 & PhacharaSuitesSukhumvit & 35 \\
\hline 0.333333333 & CondoHotelSkyyResidence & 36 \\
\hline 0.333333333 & PresidentParkBangkok & 37 \\
\hline 0.333333333 & ZenithSukhumvitHote & 38 \\
\hline 0.313953488 & CentrePointPratunam & 39 \\
\hline 0.282608696 & GracelandBangkokby & 40 \\
\hline 0.279069767 & NovotelBangkokonSi & 41 \\
\hline 0.268292683 & NovotelBangkokPlati & 42 \\
\hline 0.263888889 & AmariBoulevardBangk & 43 \\
\hline 0.235294118 & GrandMercureBangkokFortune & 44 \\
\hline 0.18 & ArnomaHotelBangkok & 45 \\
\hline 0.173913043 & GrandDiamondSuitesHotel & \\
\hline
\end{tabular}

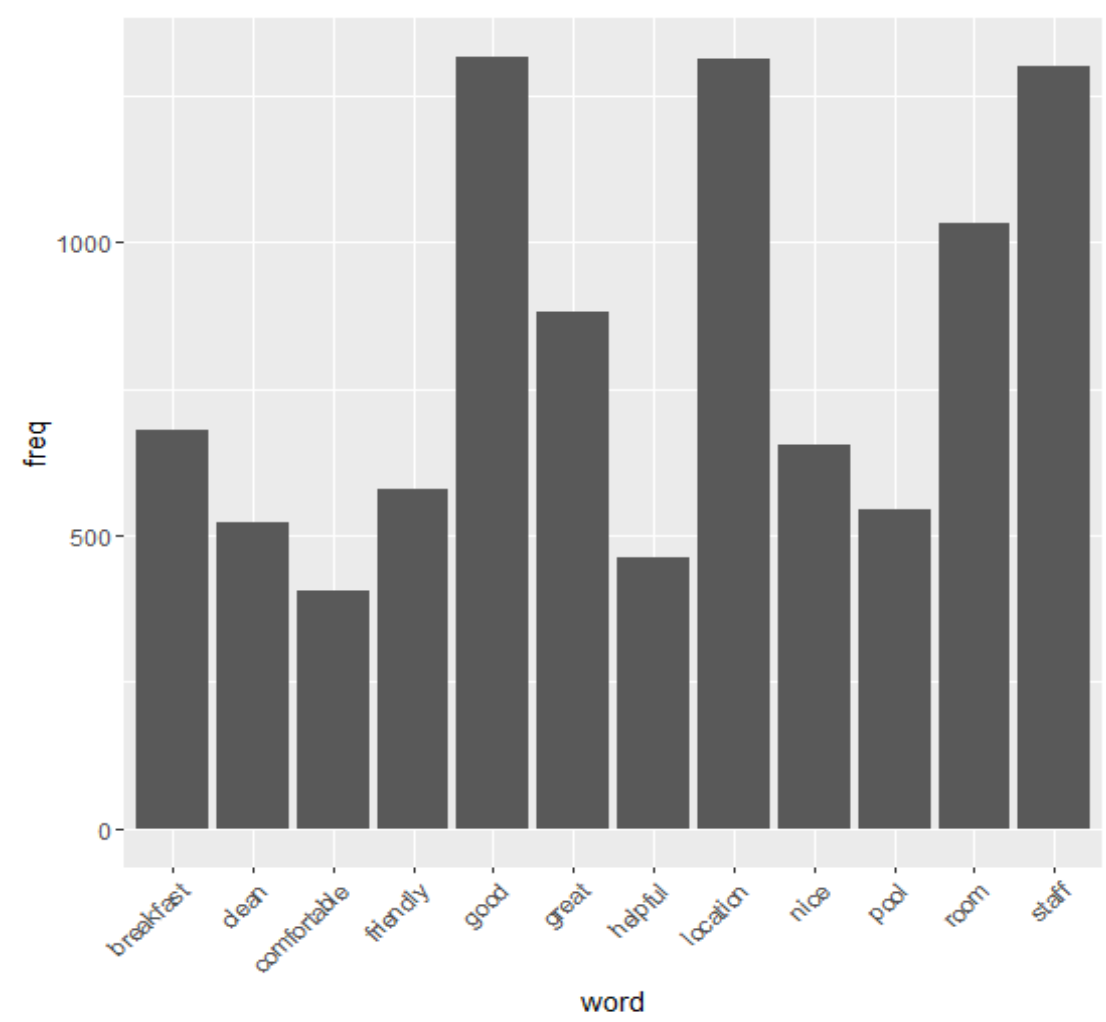

Figure1 Bangkok four Star Hotels Top Attributes 
Table 2 Ranking Five Star Hotels.

\begin{tabular}{|c|c|c|}
\hline Rank By Medium Weight & Hotel & Rank \\
\hline 0.583333333 & SivatelBangkok & 1 \\
\hline 0.567567568 & PathumwanPrincessHo & 2 \\
\hline 0.566666667 & RamadaPlazaBangkokMenamRiverside & 3 \\
\hline 0.56097561 & EastinGrandHotelSa & 4 \\
\hline 0.552631579 & InResidenceBangkokSukhumvit & 5 \\
\hline 0.551724138 & PullmanBangkokHotelG & 6 \\
\hline 0.516129032 & RadissonBluPlazaBa & 7 \\
\hline 0.515151515 & TheLandmarkBangkok & 8 \\
\hline 0.484848485 & OrientalResidenceBangkok & 9 \\
\hline 0.461538462 & FraserSuitesSukhumv & 10 \\
\hline 0.459459459 & RoyalOrchidSheratonHotelandTowers & 11 \\
\hline 0.458333333 & SwissotelLeConcordeBangkok & 12 \\
\hline 0.454545455 & SofitelBangkokSukhumvit & 13 \\
\hline 0.452380952 & TheOkuraPrestigeBangkok & 14 \\
\hline 0.447368421 & ChatriumHotelRivers & 15 \\
\hline 0.447368421 & HansarBangkok & 16 \\
\hline 0.441176471 & HotelMuseBangkokLa & 17 \\
\hline 0.428571429 & BangkokMarriottHotelSukhumvit & 18 \\
\hline 0.426086957 & GrandeCentrePointH & 19 \\
\hline 0.40625 & CentaraGrandatCent & 20 \\
\hline 0.4 & ApartmentsShamaSukh & 21 \\
\hline 0.4 & TheContinentBangkokbyCompassHospitality & 22 \\
\hline 0.393939394 & AnantaraSathornBangkokHotel & 23 \\
\hline 0.388888889 & PullmanBangkokGrandeSukhumvit & 24 \\
\hline 0.387096774 & TowerClubAtlebua & 25 \\
\hline 0.382978723 & SiriSathornExecutiveServicedResidence & 26 \\
\hline 0.382352941 & BanyanTreeBangkok & 27 \\
\hline 0.382352941 & TheSukhothaiBangkok & 28 \\
\hline 0.369565217 & RenaissanceBangkokRatchaprasongHotel,AMarriottLuxury\&LifestyleHotel & 29 \\
\hline 0.368421053 & SiamKempinskiHotel & 30 \\
\hline 0.357142857 & SwissotelNaiLertParkBangkok & 31 \\
\hline 0.35 & SOSofitelBangkok & 32 \\
\hline 0.348837209 & DreamHotelBangkok & 33 \\
\hline 0.34375 & GrandSukhumvitHotelBangkok-ManagedbyAccor & 34 \\
\hline 0.342857143 & ThePeninsulaBangkok & 35 \\
\hline 0.326086957 & ApartmentsMarriottExecutiveApartmentsMayfairBangkok & 36 \\
\hline 0.325 & CentaraGrandAtCent & 37 \\
\hline 0.3125 & AdLib & 38 \\
\hline 0.310344828 & lebuaatStateTower & 39 \\
\hline 0.305555556 & TheWestinGrandeSukhumvit,Bangkok & 40 \\
\hline 0.303030303 & NaturalVilleExecutiveResidences & 41 \\
\hline 0.266666667 & GrandHyattErawanBangkok & 42 \\
\hline 0.260869565 & CondoHotelEmporium & 43 \\
\hline
\end{tabular}




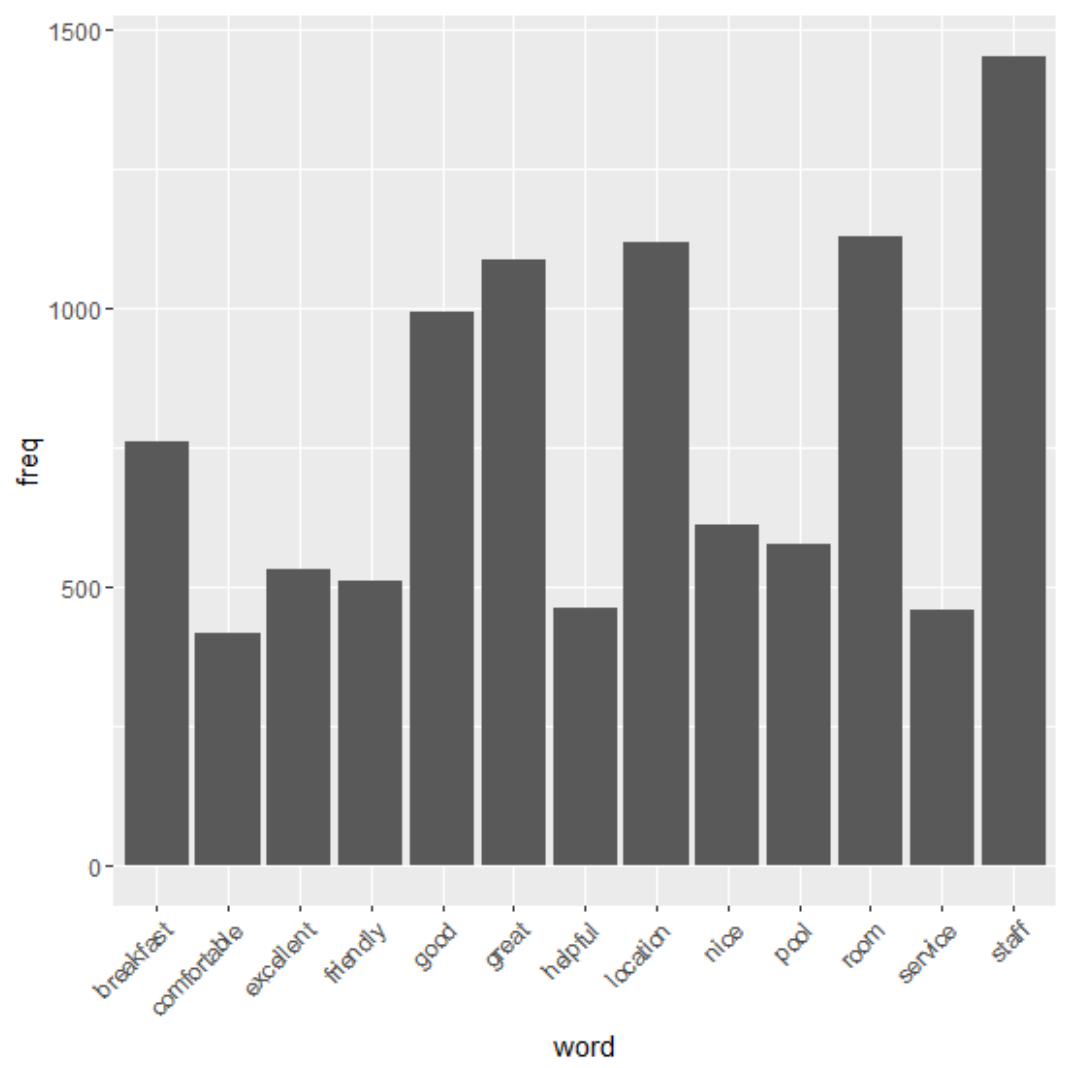

Figure 2 Bangkok five Star Hotel Top Attributes

\begin{tabular}{|c|c|c|c|c|c|c|c|c|c|}
\hline good & great & hèelpful & location & nice & pool & room & staff & Medium & Hotel \\
\hline 1 & 0.75 & 0.28571 & 0.78571 & 0.71429 & 0.57143 & 0.92857 & 0.78571 & 0.642857143 & TheDavisBangkok \\
\hline 0.93333 & 0.63333 & 0.36667 & 0.66667 & 0.56667 & 0.63333 & 0.86667 & 0.9 & 0.633333333 & FuramaSilomHotel \\
\hline 0.73333 & 0.8 & 0.36667 & 0.9 & 0.56667 & 1 & 0.66667 & 0.86667 & 0.616666667 & ApartmentsSomersetL \\
\hline 0.75 & 0.67857 & 0.53571 & 1 & 0.46429 & 0.39286 & 0.71429 & 0.96429 & 0.607142857 & NouvoCityHotel \\
\hline 0.79412 & 0.58824 & 0.29412 & 0.85294 & 0.64706 & 0.23529 & 0.61765 & 1 & 0.602941176 & MajesticGrandeHotel \\
\hline 0.78788 & 0.57576 & 0.33333 & 1 & 0.57576 & 0.45455 & 0.72727 & 0.87879 & 0.575757576 & GalleriaSukhumvit10BangkokbyCompassHospitality \\
\hline 0.67442 & 0.51163 & 0.51163 & 0.67442 & 0.5814 & 0.88372 & 0.48837 & 1 & 0.558139535 & CasaNithra \\
\hline 0.76316 & 0.44737 & 0.44737 & 1 & 0.63158 & 0.39474 & 0.65789 & 0.81579 & 0.552631579 & EvergreenPlaceBangkok \\
\hline 0.96774 & 0.77419 & 0.32258 & 1 & 0.58065 & 0.51613 & 0.90323 & 0.90323 & 0.548387097 & AloftBangkok-Sukhumvit11 \\
\hline 0.68293 & 0.53659 & 0.36585 & 1 & 0.39024 & 0.17073 & 0.58537 & 0.85366 & 0.536585366 & GrandSwissSukhumvit \\
\hline 0.62069 & 0.48276 & 0.24138 & 0.93103 & 0.41379 & 0.7931 & 0.82759 & 1 & 0.534482759 & ApartmentsSomersetS \\
\hline 0.75 & 0.61111 & 0.41667 & 0.5 & 0.52778 & 0.38889 & 0.58333 & 1 & 0.527777778 & CentrePointSukhumvi \\
\hline 0.84 & 0.44 & 0.4 & 0.68 & 0.36 & 0.36 & 0.96 & 1 & 0.52 & ApartmentsSomersetP \\
\hline 0.94118 & 0.5 & 0.14706 & 1 & 0.64706 & 0.41176 & 0.52941 & 0.73529 & 0.514705882 & DynastyGrandeHotel \\
\hline 0.875 & 0.375 & 0.28125 & 0.9375 & 0.34375 & 0.40625 & 1 & 1 & 0.5 & CentrePointSilom \\
\hline 0.55556 & 0.55556 & 0.36111 & 0.75 & 0.44444 & 0.44444 & 0.55556 & 1 & 0.5 & ParkPlazaSukhumvit \\
\hline 1 & 0.475 & 0.425 & 0.8 & 0.45 & 0.15 & 0.875 & 0.9 & 0.4875 & CentrePointChidlom \\
\hline 0.67742 & 0.54839 & 0.16129 & 1 & 0.16129 & 0.41935 & 0.90323 & 0.6129 & 0.483870968 & MercureBangkokSiam \\
\hline 0.52 & 0.5 & 0.32 & 0.34 & 0.46 & 0.56 & 0.48 & 1 & 0.47 & USukhumvitBangkok \\
\hline 0.72093 & 0.5814 & 0.37209 & 1 & 0.2093 & 0.2093 & 0.44186 & 0.83721 & 0.465116279 & AdelphiSuitesBangkok \\
\hline 0.67647 & 0.44118 & 0.26471 & 0.82353 & 0.41176 & 0.32353 & 0.47059 & 1 & 0.455882353 & BestWesternPremier \\
\hline 0.54054 & 0.27027 & 0.2973 & 0.48649 & 0.45946 & 0.51351 & 0.43243 & 1 & 0.445945946 & ApartmentsLohasSUITESSUKHUMVIT bySUPERHOTEL \\
\hline 0.69697 & 0.33333 & 0.33333 & 1 & 0.33333 & 0 & 0.63636 & 0.54545 & 0.439393939 & HolidaylnnExpressBangkokSiam \\
\hline 0.61538 & 0.71795 & 0.28205 & 0.07692 & 0.41026 & 1 & 0.46154 & 0.92308 & 0.435897436 & ChatriumResidenceSathonBangkok \\
\hline 0.68 & 0.64 & 0.24 & 1 & 0.2 & 0.2 & 0.72 & 0.76 & 0.42 & BaiyokeSkyHotel \\
\hline 0.58333 & 0.52778 & 0.16667 & 1 & 0.27778 & 0.41667 & 0.55556 & 0.72222 & 0.402777778 & RembrandtHotelBangkok \\
\hline 0.84848 & 0.27273 & 0.42424 & 0.81818 & 0.33333 & 0.33333 & 0.87879 & $\overline{1}$ & 0.393939394 & PresidentSolitaireHotel\&Spa \\
\hline 0.71875 & 0.0625 & 0.125 & 1 & 0.21875 & 0.125 & 0.4375 & 0.71875 & 0.390625 & GMSuites \\
\hline 0.3 & 0.72 & 0.44 & 0.7 & 0.4 & 0.58 & 0.38 & 1 & 0.39 & RivaSuryaBangkok \\
\hline 0.58974 & 0.53846 & 0.33333 & & 0.20513 & 0.30769 & 0.58974 & 0.87179 & 0.384615385 & FourPoints bySheratonBangkok,Sukhumvit15 \\
\hline
\end{tabular}

Figure 3 four star hotels sample ranking 


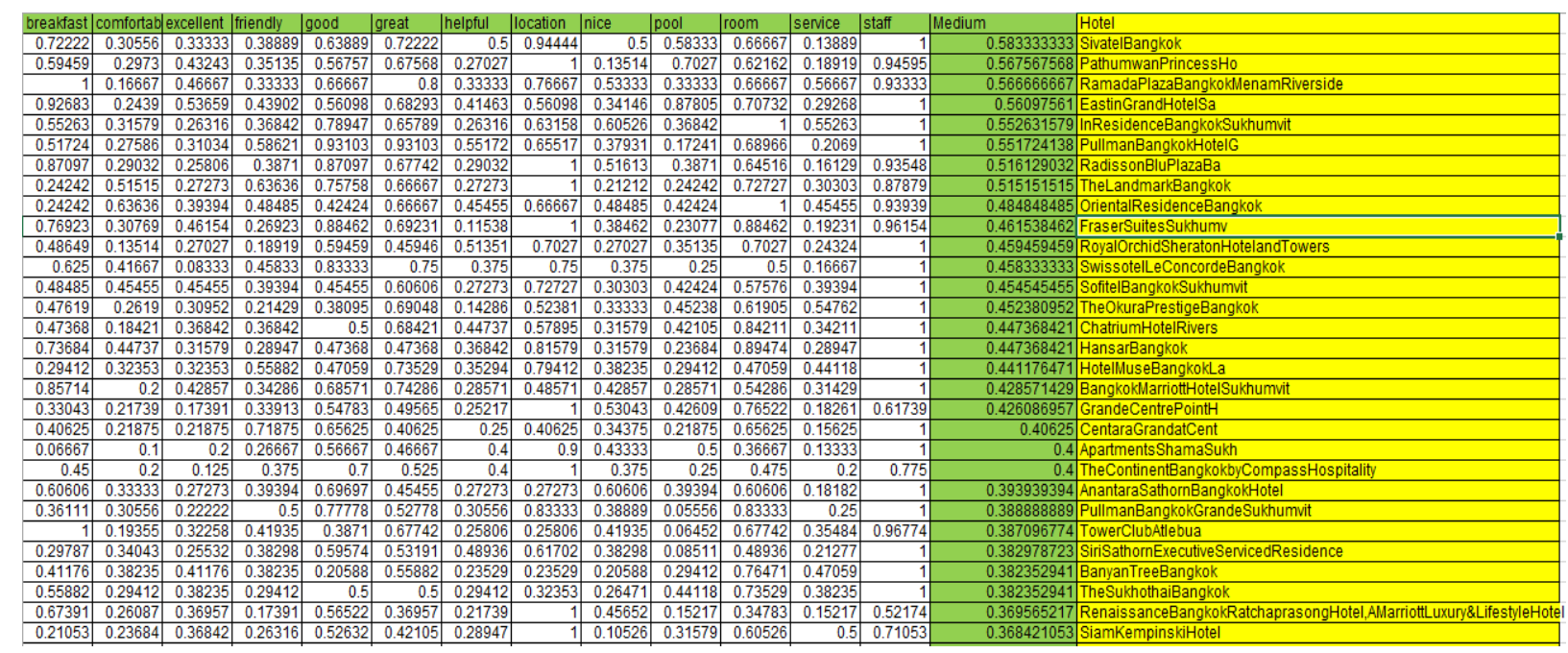

Figure 4 five star hotels sample ranking 


\section{DISCUSSION}

Based on above results, some attributes are discovered as top attributes that are shown on figure1 and figure2, these attributes are selected based on minimum word frequencies and these minimum choose per words repeat and it's 400 that help us to decrease the scale of attributes and increase the confidence of result then two ranking tables are provided with word frequency for each attribute and each hotel and two tables are created for four and five star hotels. As results show that three attributes such as staff, location and room have more weight for determining high rank hotels but high weight in five hotels result belongs to staff but in four star hotels each mentioned attributes has been distributed normally between hotels and the lower attribute for four star hotels belongs to "comfortable" and for five star hotels belongs to "service"

\section{CONCLUSION}

As this paper mentioned, choosing a good hotel is one of major problem for each tourism that want to travel to abroad so based on mentioned method which has been provided we have ranked all hotels based on important and top attributes which are shown on table1 and table 2 that guide us to choose a good hotel meanwhile it helps to owner of hotels to fix their problems in some attributes that they have lower score also new customer can decide to choose better hotels per results. At the final our suggestion for future works is to provide a suggestion and decision system for introduce top hotels with specific attribute, it means a customer choose some attribute and system suggest top hotels with mentioned attributes and rank based on weight and attributes score.

\section{REFERENCES}

Ariffin, A. A. M., \& Maghzi, A. (2012). A preliminary study on customer expectations of hotel hospitality: influences of personal and hotel factors. International Journal of Hospitality Management, 31(1), 191e198.

Atkinson, A. (1988). Answering the eternal question: what does the customer want? The Cornell Hotel and Restaurant Administration Quarterly, 29(2), 12e14.
Baumgarten, J. C., \& Kent, G. J. W. (2010). Executive summary: Travel and tourism economic impact. London, United Kingdom: World Travel and Tourism Council.K. Elissa, "Title of paper if known," unpublished.

Browning, V., So, K.K.F., Sparks, B., 2013. The influence of online reviews on con-sumers' attributions of service quality and control for service standards in hotels.J. Travel Tour. Mark. 30, 23-40.

Buhalis, D., \& Law, R. (2008). Progress in information technology and tourism management: 20 years on and 10 years after the Internet the state of eTourism research. Tourism Management, 29(4), $609 \mathrm{e} 623$

Bulchand-Gidumal, J., Melian-Gonzalez, S., \& Lopez-Valcarcel, B. G. (2011). Improving hotel ratings by offering freeWi-Fi. Journal of Hospitality and Tourism Technology, 2(3), 235e245.

Chen, C.-F., Chen, F.-S., 2010. Experience quality, perceived value, satisfaction andbehavioral intentions for heritage tourists. Tourism Manage. 32, 29-35.

Clemes, M.D., Gan, C., Ren, M., 2011. Synthesizing the effects of service quality, value, and customer satisfaction on behavioral intentions in the motel industry: an empirical analysis. J. Hosp. Tourism Res. $35,530-568$.

Dolnicar, S. (2002). Business travelers' hotel expectations and disappointments: a different perspective to hotel attribute importance investigation. Asia Pacific Journal of Tourism Research, 7(1), 29e35.

Engel, J.F., Blackwell, R.D., Miniard, P.W., 1990. Consumer Behavior, 6th ed. Dryden Press, Hinsdale, IL.

Gretzel, U., Yoo, K. H., \& Purifoy, M. (2007). Online travel reviews study: Role and impact of online travel reviews. A\&M University, Texas: Laboratory for Intelligent Systems in Tourism. Retrieved April 2, 2015

from http://195.130.87.21:8080/dspace/bitstream/1234567 89/877/1/Online\%20travel\%20review\%20study\%20r ole $\% 20$ and $\% 20$ impact $\% 20$ of $\% 20$ online $\% 20$.pdf.

Hennig-Thurau, T., Gwinner, K. P., Walsh, G., \& Gremler, D. D. (2004). Electronic word-of-mouth via consumer-opinion platforms: what motivates consumers to articulate themselves on the internet? Journal of Interactive Marketing, 18(1),38e52.

Hunt, J.D., 1975. Image as a factor in tourism development. J. Travel Res. 13, 3-7.

Keates, N. (2007, June 1). Deconstructing TripAdvisor. The Wall Street Journal. Retrieved April 17, 2015 from http://online.wsj.com/article/SB11806556911692071 0.html. 
Kotler, P., Bowen, J.T., Makens, J.C., 2006. Marketing for Hospitality and Tourism. Pearson Education, India.

Lai, L. H., \& Graefe, A. R. (2000). Identifying market potential and destination choice factors of Taiwanese overseas travelers. Journal of Hospitality and Leisure Marketing, 6(4), 45e65.

Lee, C. F., Huang, H. I., \& Chen, W. C. (2010). The determinants of honeymoon destination choice the case of Taiwan. Journal of Travel and Tourism Marketing, 27(7), 676e693.

Lewis, R. C. (1985). Getting the most from marketing research (part V). Predicting hotel choice: the factors underlying perception. The Cornell Hotel and Restaurant Administration Quarterly, 25(4), 82e96.

Meyer, C., Schwager, A., 2007. Understanding customer experience. Harvard Bus.Rev. (February), 117-126.
Namasivayam, K., Enz, C. A., \& Siguaw, J. A. (2000). How wired are we? The selection and use of new technology in US Hotels. The Cornell Hotel and Restaurant Administration Quarterly, 41(6), 40e48.

Oh, H., Parks, S.C., 1997. Customer satisfaction and service quality: a critical review of the literature and research implications for the hospitality industry. Hosp.Res. J. 20, 35-64.

Oliver, R.L., 1981. Measurement and evaluation of satisfaction processes in retail settings. J. Retail. 57, 25-48.

Page, S., \& Connell, J. (2006). Tourism: A modern synthesis. London: Thomson.

Poston, R. S. (2008). Using and fixing biased rating schemes. Communications of the ACM, 51(9), $105 \mathrm{e} 109$.

Rivers, M. J., Toh, R. S., \& Alaoui, M. (1991). Frequent-stayer programs: The demographic, behavioral, and attitudinal characteristics of hotel steady sleepers. Journal of Travel Research, 30(2), $41 \mathrm{e} 45$.

Wood, S.A., Guerry, A.D., Silver, J.M., Lacayo, M., 2013. Using social media to quantify nature-based tourism and recreation. Sci. Rep. 3, 2976. 